\title{
DÜBLIN
}

Technological University Dublin

ARROW@TU Dublin

\section{Development of Photopolymer Material with Improved Dynamic Range and Sensitivity}

\author{
Brian Rogers \\ c14507363@mytudublin.ie \\ Izabela Naydenova \\ Technological University Dublin, izabela.naydenova@tudublin.ie \\ Suzanne Martin \\ suzanne.martin@tudublin.ie
}

Follow this and additional works at: https://arrow.tudublin.ie/scschphycon

Part of the Optics Commons

\section{Recommended Citation}

Rogers, B., Naydenova, I. \& Martin, S. (2019). Development of photopolymer material with improved dynamic range and sensitivity. SPIE Nanoscience + Engineering, 2019, Proceedings Active Photonic Platforms, vol. 11081. https://doi.org/10.1117/12.2528715

This Conference Paper is brought to you for free and open access by the School of Physics \& Clinical \& Optometric Science at ARROW@TU Dublin. It has been accepted for inclusion in Conference Papers by an authorized administrator of ARROW@TU Dublin. For more information, please contact arrow.admin@tudublin.ie, aisling.coyne@tudublin.ie, gerard.connolly@tudublin.ie. Funder: TU Dublin 


\section{Development of photopolymer material with improved dynamic range and sensitivity}

Brian Rogers, Izabela Naydenova, Suzanne Martin

Brian Rogers, Izabela Naydenova, Suzanne Martin, "Development of photopolymer material with improved dynamic range and sensitivity," Proc.

SPIE 11081, Active Photonic Platforms XI, 110812G (5 September 2019); doi: 10.1117/12.2528715

SPIE Event: SPIE Nanoscience + Engineering, 2019, San Diego, California, United States 


\title{
Development of Photopolymer Material with Improved Dynamic Range and Sensitivity
}

\author{
Brian Rogers, Suzanne Martin, Izabela Naydenova
}

Dublin Institute of Technology, Ireland

\begin{abstract}
:
In this study the effect of the concentration of acrylamide and the influence of different initiators in a photopolymer composition for holographic recording of diffraction gratings is investigated. Light manipulating Holographic Optical Elements (HOEs) have a number of characteristics which can be optimised for different roles. However, at the core of these devices is the refractive index modulation that has been created in the material during recording. Typically higher refractive index modulation will enable greater diffraction efficiency. Solar concentrating HOEs can particularly benefit from material that experiences higher refractive index modulation. For a solar concentrator to have a high acceptance angle thinner photopolymer layers are preferable. Thinner layers can lead to a reduction of the device's diffraction efficiency unless the refractive index modulation increases to compensate.

This paper presents an optimisation of a photopolymer formulation for improved refractive index modulation and sensitivity of the layer. An increase in the acrylamide concentration of $66 \%$ resulted in $50 \%$ higher refractive index modulation with values reaching $5 \times 10^{-3}$ in 40 micron layers.

Faster recording times are an important consideration for the commercialization and mass production of photopolymer devices [1]. Higher production rates and lower costs are some of the main advantages. Altering the initiator is expected to have an effect on the material's sensitivity and thus on recording time.

Several initiators were compared, triethanolamine (TEA), methyldiethanolamine (MDEA) and dimethylethanolamine (DMEA). It was found that holograms recorded with MDEA as the initiator recorded 58\% faster over TEA based photopolymer at larger layer thicknesses.

The stability of the photopolymer was also tested with different protective coatings when subjected to UV light. The properties exhibited by this photopolymer composition make it a promising candidate for development of solar concentrating applications; however improvements to the durability in conditions of UV radiation will have to be made before it is suitable for solar concentration.
\end{abstract}

Active Photonic Platforms XI, edited by Ganapathi S. Subramania, Stavroula Foteinopoulou,

Proc. of SPIE Vol. 11081, 110812G · C 2019 SPIE · CCC code: 0277-786X/19/\$21

doi: $10.1117 / 12.2528715$

Proc. of SPIE Vol. 11081 110812G-1

Downloaded From: https://www.spiedigitallibrary.org/conference-proceedings-of-spie on 10 Sep 2019 Terms of Use: https://www.spiedigitallibrary.org/terms-of-use 


\section{Introduction:}

Holographic devices recorded using photopolymer are diffractive devices capable of fulfilling the role of a number of refractive optical devices such as lenses, beam splitters and mirrors [2]. These devices have a number of advantages over their traditional refraction based counterparts. They are lightweight, only a few tens of microns thick and are capable of fulfilling their device role with near $100 \%$ efficiency. They are also mass-producible and low-cost making them ideal for mass manufacturing [1].

Photopolymers still face a number of challenges when it comes to optimising certain devices and mass production. Solar concentrating holographic devices should ideally have a low angular selectivity and a high durability, this allows for solar concentration at any angle without the use of solar tracking, and reduces the need to be replaced as frequently. To achieve this, a high dynamic range photopolymer material capable of withstanding constant UV radiation and temperature variations is required. The sensitivity of the material is also an important consideration when it comes to mass production. The faster the holographic device can be recorded the larger the potential output of devices.

The chemical composition of the photopolymer material is responsible for its recording properties. Changing the ratios of one compound to another can have significant effects on the photopolymer's performance. This work looks to optimise the chemical composition of the photopolymer in order to achieve the best preforming formulation. Two aspects of the photopolymer were changed, the monomer concentration and the initiator molecule. Both the monomer and initiator were previously found to have effects on the dynamic range and sensitivity of the photopolymer [3][4].

Changes to the concentration of the acrylamide monomer were made; this was found to have effects on both the recording time and the final refractive index modulation. An optimal concentration was determined in order to maximise both recording time and refractive index modulation.

Three initiator molecules were investigated, triethanolamine (TEA), methyldiethanolamine (MDEA) and dimethylethanolamine (DMEA). Previous studies show that the initiator molecule had effects on the bleaching rates of photopolymer [3]. MDEA was shown to have a faster bleaching time than TEA. In this study we aim to show that this faster bleaching rate revealing a faster interaction of the dye molecules with the initiator can extend to holographic recording. DMEA was also included in this study for its chemical similarity to TEA and MDEA as shown in the figure below.

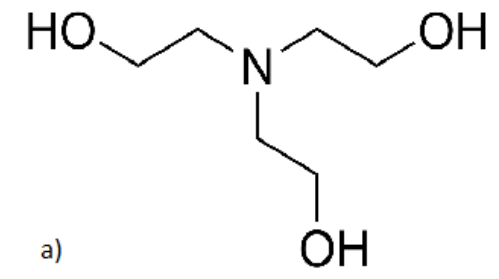

b)

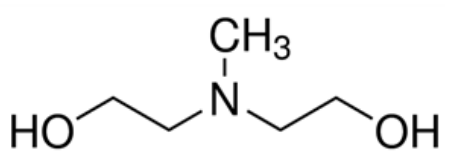

Figure 1: Structure of a) TEA, b) MDEA and c) DMEA.

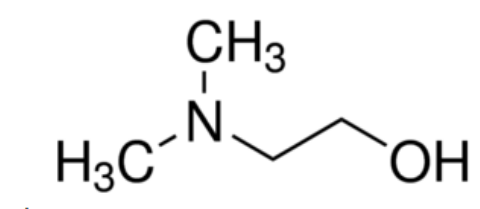

c) 


\section{Theory:}

2.1 Properties of Photopolymer:

Photopolymer is a photosensitive material which polymerises in the presence of light with suitable energy. There are several components of the photopolymer which make this possible. Photopolymer consists of a binder, dye, initiator, monomer and cross-linker. The dye absorbs energy from the light and enters an elevated energy state, in this state it interacts with the initiator molecule and thus producing a free radical. This free radical then interacts with a monomer molecule which will in turn interact with more monomers to form a polymer chain. The cross linker allows large polymer chains to interact and form a single larger chain. Its main role is to immobilise the produced photopolymer chains and thus make them more stable. The binder matrix stops the polymer chains from diffusing once they have formed. Figure 2 below shows how this process occurs.

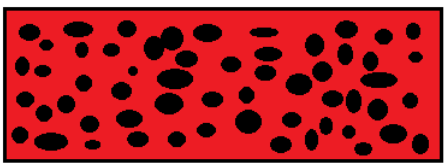

Monomer randomly dispersed

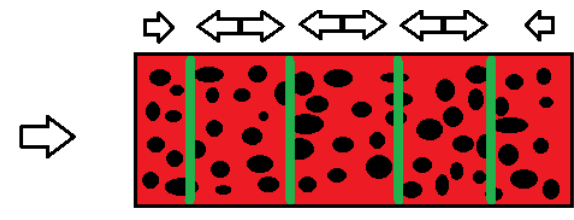

Monomer diffusion occurs. Monomer reacts in illuminated areas.
Photopolymer illuminated with interference pattern
Photopolymer illuminated for up to one minute

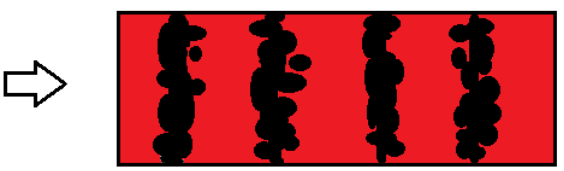

Monomer fully diffused and polymerised in illuminated areas.

Figure 2: Photopolymer reaction process.

\subsection{Regimes of operation of holographic gratings:}

Holograms can operate in different regimes depending on the thickness, refractive index modulation and fringe spacing of the hologram. There are 2 main modes of operation, as thin and thick holograms. In solar concentrating holograms it is most common to work in the regime of thick holograms. This is because only thick holograms can achieve 100\% diffraction efficiency in sinusoidal gratings. The Q-factor of a hologram is one method of determining what regime one is working in, it is described as follows[5]:

$$
Q=\frac{2 \pi \lambda d}{n_{0} \Lambda^{2}}(1)
$$

Where $\lambda$ is the recording wavelength, $\mathrm{d}$ is the thickness, $n_{0}$ is the refractive index and $\Lambda$ is the fringe spacing. In general $Q$ values greater than 10 are considered to be in the thick regime while values less than 1 are considered thin.

Hologram thickness however affects the angular selectivity of the device. Thicker holograms are more angularly selective, to achieve less angularly selective holograms thinner holograms must be used and so the refractive index modulation must increase to compensate for the loss in diffraction efficiency. The angular selectivity is also dependant on the fringe spacing.

The angular selectivity follows the form of a Bragg curve as in figure $3 a$ ) below. For unslanted gratings, the angle between the peak and minima can be given by equation 2 .

$$
\Delta \theta \approx \frac{\Lambda}{\mathrm{d}}(2)
$$

Where $\mathrm{d}$ is the thickness and $\Lambda$ is the fringe spacing.

For solar concentrating devices a balance must be kept to stay in the thick regime but also have a small angular selectivity. 


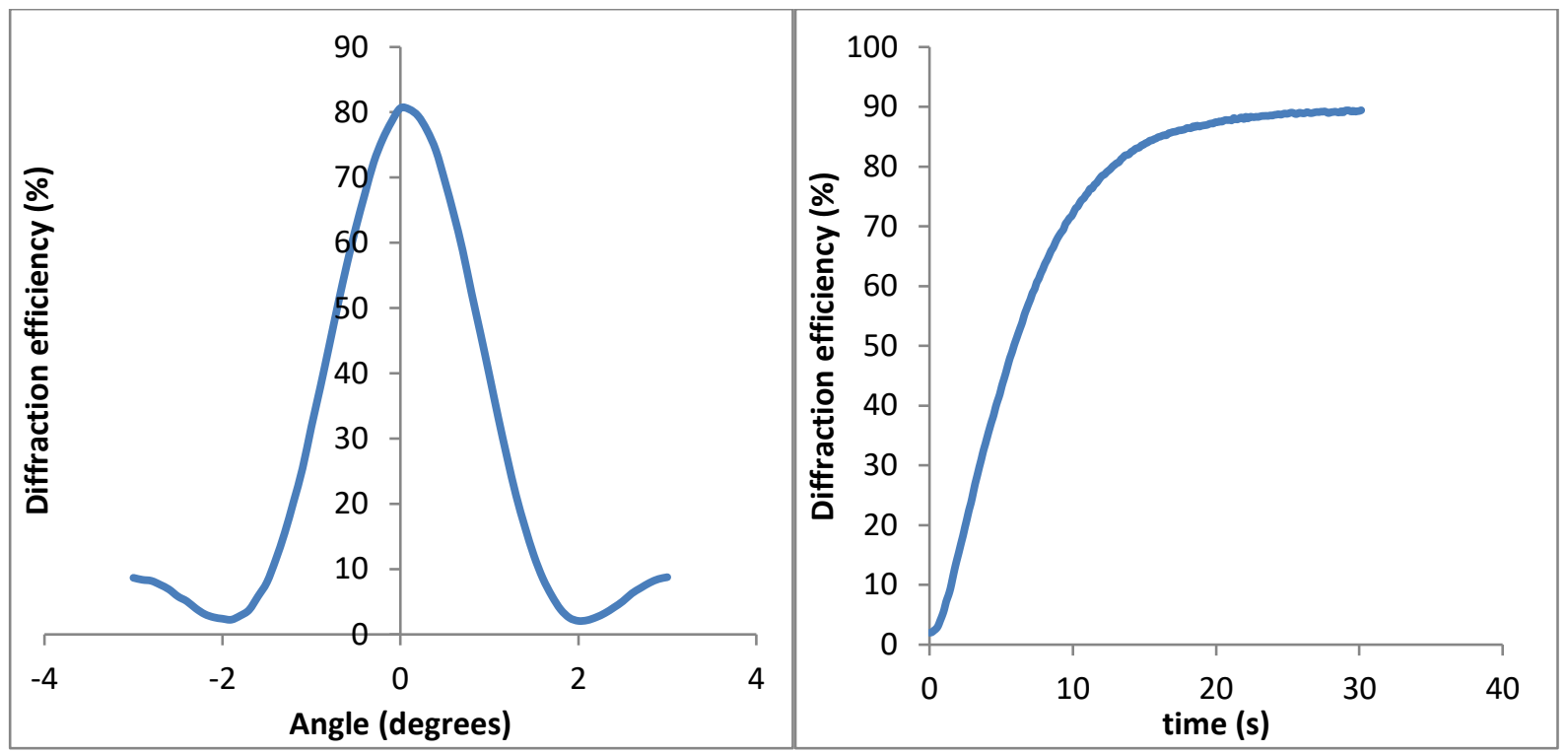

Figure 3: a) Example Bragg curve (left) and b) real time growth curve (right). Both samples were $45 \pm 5$ microns thick recorded with a combined beam intensity of $5 \mathrm{mW/ \textrm {cm } ^ { 2 }}$.

In order to experimentally determine the refractive index modulation of the hologram Kogelnik's theory [6] shown in equation 3 below was used.

$$
\Delta n=\frac{\sin ^{-1} \sqrt{\eta} \cos (\theta) \lambda}{\pi d}(3)
$$

Where $\Delta n$ is the refractive index modulation, $\theta$ is the Bragg angle of incidence of the probe laser beams, $\lambda$ is the wavelength of the probing laser beam, $\mathrm{d}$ is the thickness of the photopolymer layer and $\eta$ is the diffraction efficiency.

Equation 2 can be used to find the thickness from the Bragg curve of a hologram. The diffraction efficiency can be found in real time recordings of the grating formation as in the example below.

When determining rate of grating formation the slope of the linear section of the graph was used. For example, in figure $3 b$ ) above the time between 1 and 6 seconds was used.

\section{Materials and methods:}

\subsection{Materials}

Several photopolymer solutions were prepared using various concentrations of acrylamide.

First a PVA stocksolution (9\% wt) was prepared by dissolving $10 \mathrm{~g}$ of PVA in $100 \mathrm{ml}$ of deionized water under constant stirring at $50^{\circ} \mathrm{C}$. The solution was then poured through filter paper. A dye stock solution was also prepared by dissolving $0.11 \mathrm{~g}$ of Erythrosine $\mathrm{B}$ in $100 \mathrm{ml}$ of deionized water.

The photopolymer was prepared by mixing $4.38 \mathrm{ml}$ of the PVA solution, $1 \mathrm{ml}$ of the dye solution, $0.5 \mathrm{ml}$ of TEA initiator, $0.05 \mathrm{~g}$ of N, N' Methylene bis-acrylamide cross-linker and acrylamide. Four masses of acrylamide were used to prepare different solution concentrations, $0.15 \mathrm{~g}, 0.20 \mathrm{~g}, 0.25 \mathrm{~g}$, and $0.3 \mathrm{~g}$. These masses produced solutions with an acrylamide concentration of $0.355,0.474,0.592$ and $0.711 \mathrm{M}$ respectively. 
Extensive previous studies investigate formulation which uses $0.15 \mathrm{~g}$ of acrylamide [4]. All changes are compared to this previously studied formulation..

A pipette was used to spread between $300 \mathrm{~mL}-500 \mathrm{~mL}$ depending on the desired thickness of photopolymer onto glass microscope slides with an area of $75 \times 25 \mathrm{~mm}^{2}$. The samples were left to dry overnight.

For the initiator tests the photopolymer was prepared in the same way as outlined above, using the optimal acrylamide concentration. In order to keep the concentration of initiator in moles the same in each solution $0.430 \mathrm{~mL}$ of MDEA and $0.375 \mathrm{~mL}$ of DMEA was used. Figure 4 below shows how keeping the initiators equal molar concentration effects the mass distribution of each compound in the photopolymer.

The samples were prepared identically to the previous section, using a pipette to spread $300 \mathrm{~mL}-500 \mathrm{~mL}$ of solution onto a microscope slide.

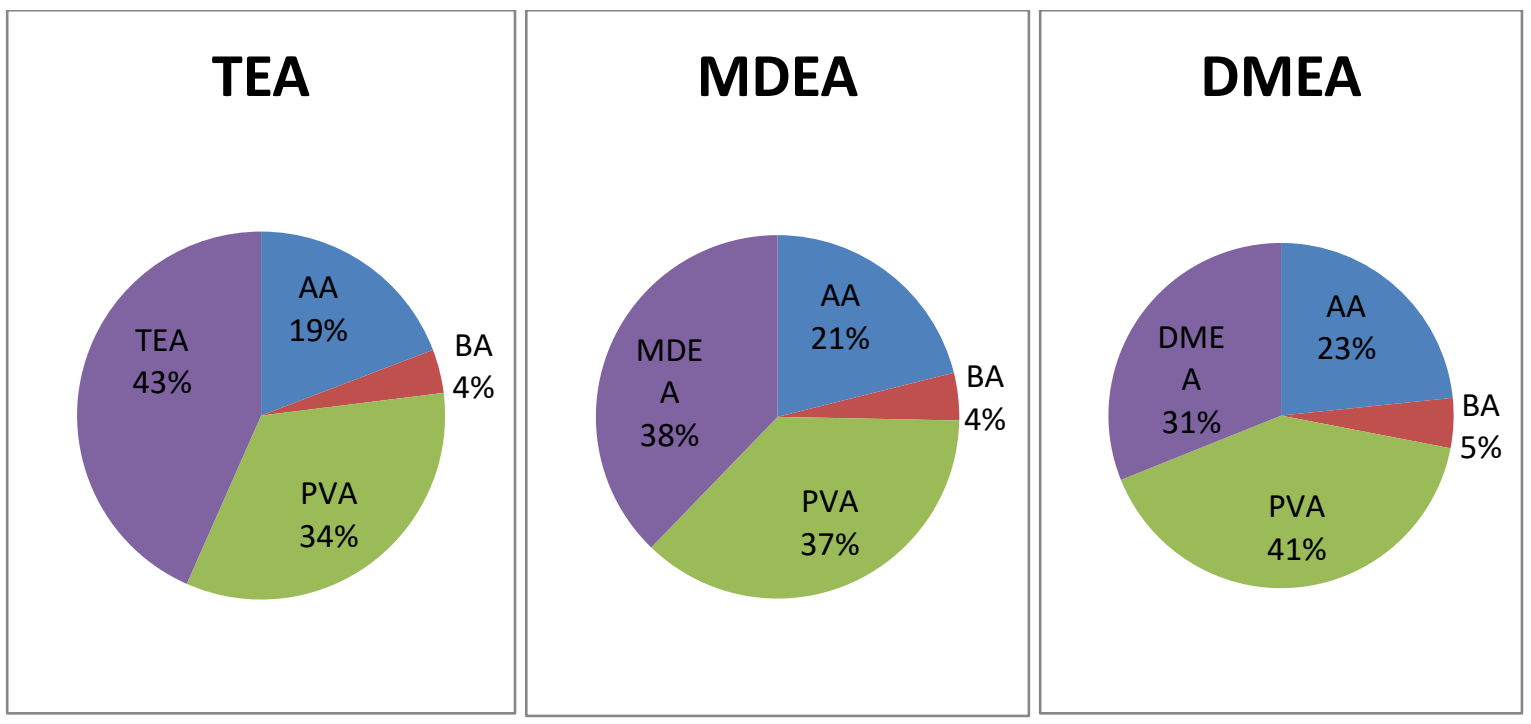

Figure 4: Mass distribution of all materials used to investigate the effect of initiator when initiators are equal concentration in moles.

\subsection{Methods:}

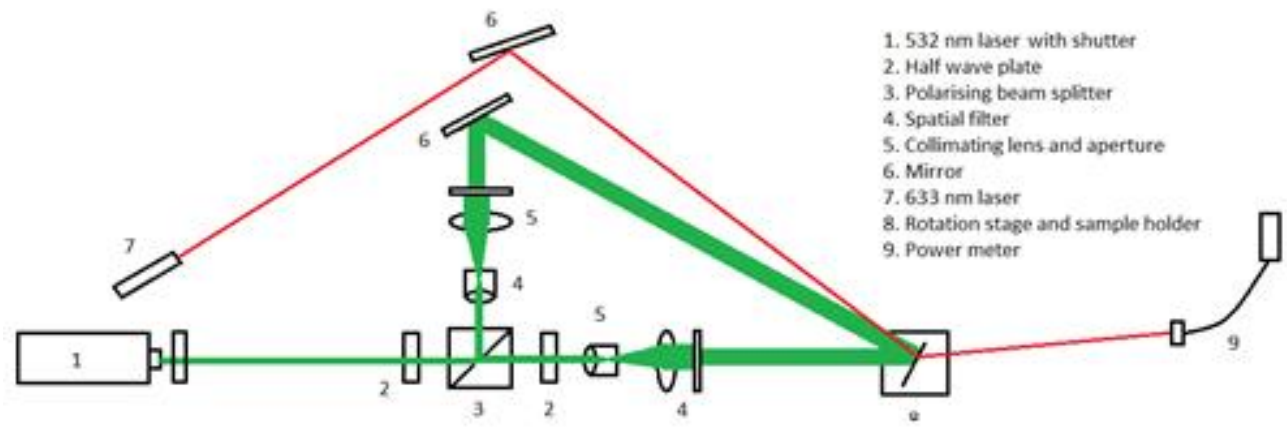

Figure 5: Holographic recording and real time monitoring set up.

The set up in figure 5 was used to record volume diffraction gratings in the samples. After opening a shutter the 532 $\mathrm{nm}$ beam passes through a half wave plate followed by a polarising beam splitter. One beam is spatially filtered, collimated and directed towards the sample using a mirror. The other beam travels through another half wave plate, 
then spatially filtered and collimated. This system allows the intensity of both beams to be controlled and made equal. The two beams meet at the sample where holographic recording takes place. The $633 \mathrm{~nm}$ probe beam is incident on Bragg angle and the beam diffracted in the first order of diffraction is incident on a power meter where the grating formation is tracked in real time using a computer. The samples were recorded at $800 \mathrm{lines} / \mathrm{mm}$ with a combined beam intensity of approximately $5 \mathrm{~mW} / \mathrm{cm}^{2}$. After the samples were recorded a Bragg curve of the hologram was taken using a computer controlled rotating stage on which the sample was placed.

\section{Results:}

4.1 Refractive index modulation comparison:

Before the refractive index modulation photoinduced in layer of different initiators was compared, the concentration of acrylamide was optimised in TEA based photopolymer. Figure 6 below shows the dependence of refractive index modulation on acrylamide concentration.

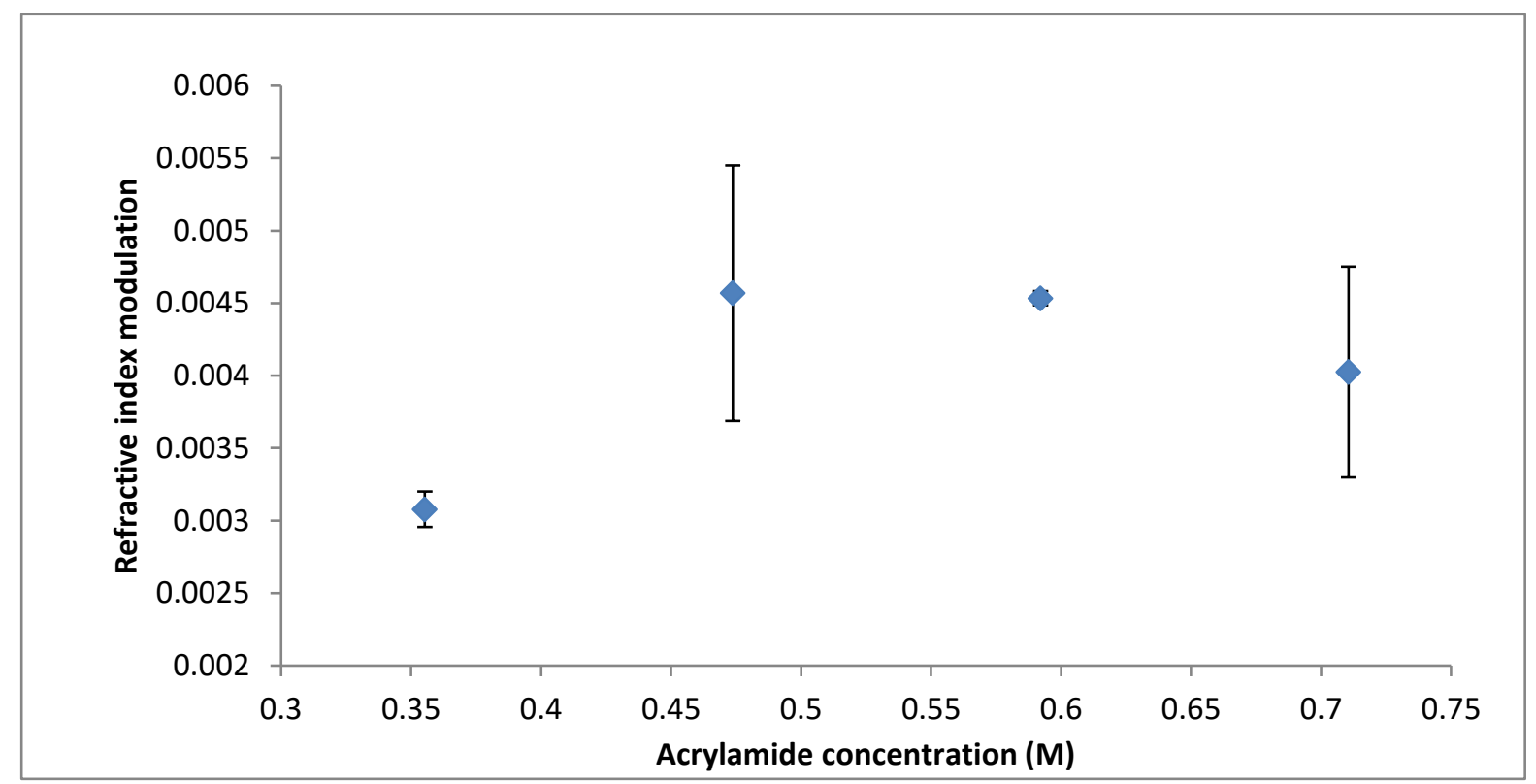

Figure 6: Refractive index modulation vs acrylamide concentration in $45 \pm 5$ micron thick layers with a recording intensity of 5 $\mathrm{mW} / \mathrm{cm}^{2}$

An acrylamide concentration of $0.59 \mathrm{M}$ proved to have the largest refractive index modulation on average. This is the concentration used while comparing the effects of the initiators.

4.2 Initiator comparison at different thicknesses:

Firstly it is important to note the quality of the photopolymer layers when prepared with different initiators. TEA and MDEA layers dried normally and formed uniform layers. DMEA however, due to its high vaper pressure, evaporated and formed non-uniform layers capable of only limited holographic recording. Therefore only TEA and MDEA will be compared.

A comparison between the refractive index modulation was made at several different thicknesses in TEA and MDEA based photopolymer. These can be compared in the figure below: 


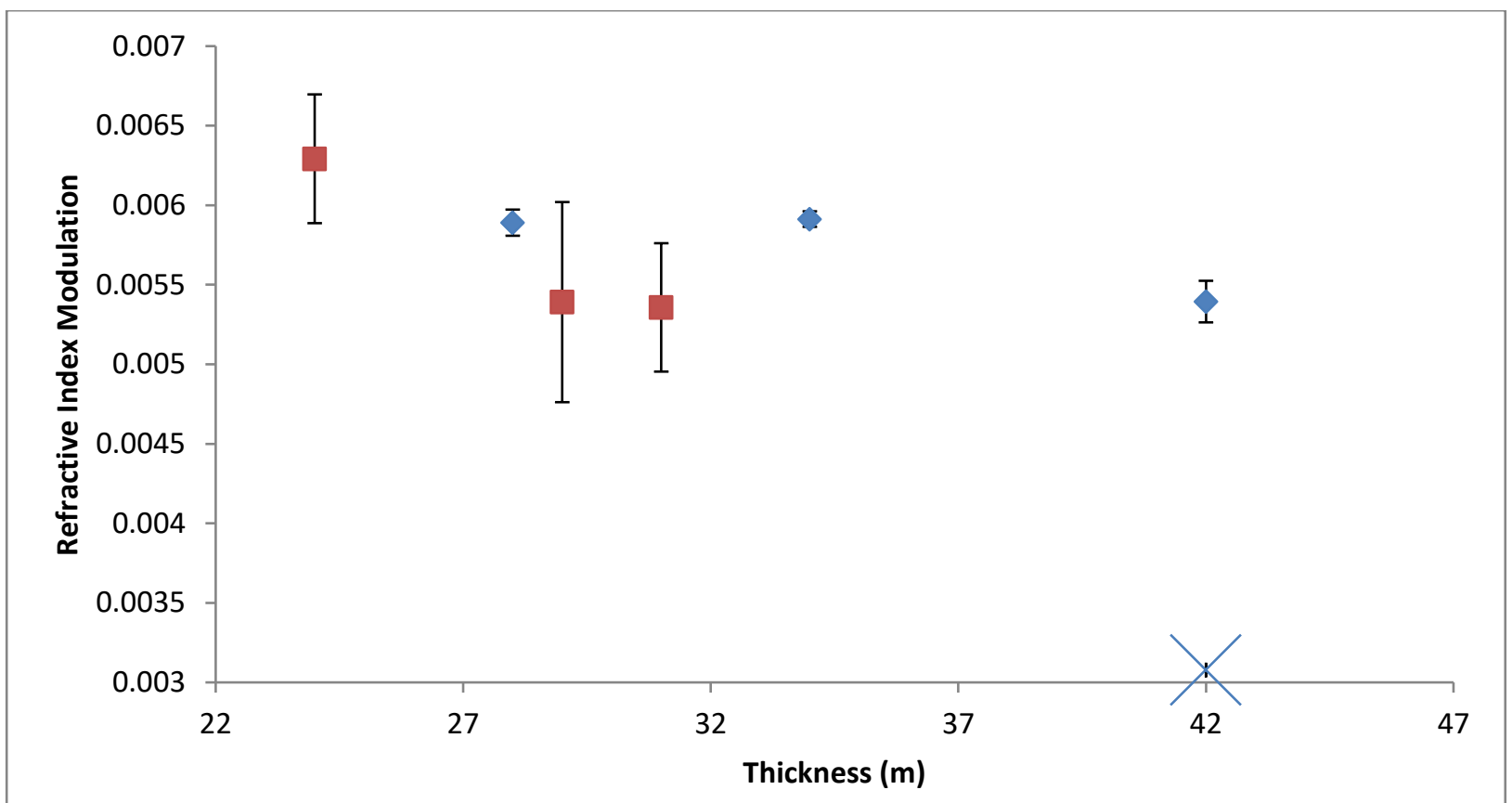

Figure 7: Refractive index modulation at different thicknesses with TEA (red) and MDEA (blue) as initiator.

Figure 7 shows there is little difference in refractive index modulation between initiators (within the experimental error), this is true across a range of thicknesses. On average the MDEA based photopolymer has a $2.7 \%$ higher refractive index modulation.

This similarity in refractive index modulation makes MDEA as viable as TEA as an initiator with the added bonus of increased growth rates at higher thicknesses. The point marked by the $\mathrm{X}$ shows the refractive index modulation of the photopolymer before acrylamide optimisation in TEA based photopolymer at a thickness of 42 microns.

\subsection{Grating formation rates:}

The rate of formation of holographic gratings was determined at different thicknesses of photopolymer. The difference in this rate can be seen in figure 8 below. The point marked by the $\mathrm{X}$ shows the original formulation's rate of formation at a thickness of 42 microns before any acrylamide optimisation. 


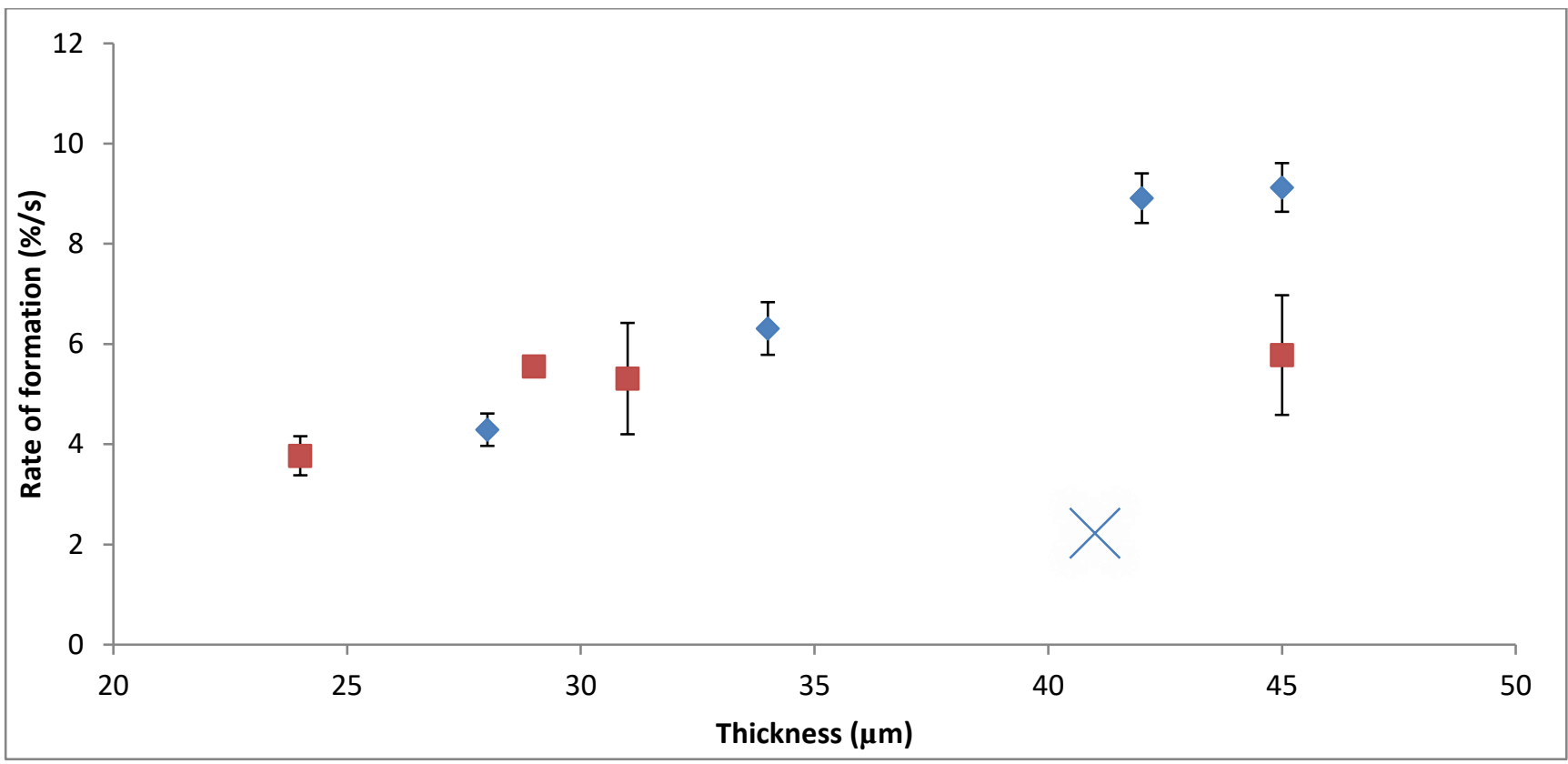

Figure 8: Rate of formation of holographic gratings using TEA (red) and MDEA (blue) as initiator.

When looking at the rate of change of grating formation rates across thicknesses, MDEA based photopolymer's growths rates increase 4 times more than TEA based photopolymer does as thickness increases. This means MDEA based photopolymer performs similarly to TEA at low thicknesses, however, the thicker the MDEA based photopolymer is the better it will perform in comparison to TEA. This is likely due to the rate of diffusion of the molecule; MDEA is a smaller molecule with fewer hydrogen bonds than TEA, this would make MDEA more free to move than TEA. This increased diffusion could be responsible for the increased formation rates.

\subsection{Preliminary UV results:}

Samples made with the optimal concentration of both acrylamide and MDEA were subject to UVA radiation of 60 $\mathrm{mW} / \mathrm{cm}^{2}$. Figure 9 shows the degradation caused by the radiation on a hologram coated with UV protective plastic after 40 hours of exposure. The broadening of the Bragg curve and decrease in diffraction efficiency would imply that the UV radiation caused shrinkage in the layers. The shrinkage reduces the effective thickness of the hologram; thickness affects both the diffraction efficiency and angular selectivity, since the thickness is reduced both the diffraction efficiency and angular selectivity are reduced. 


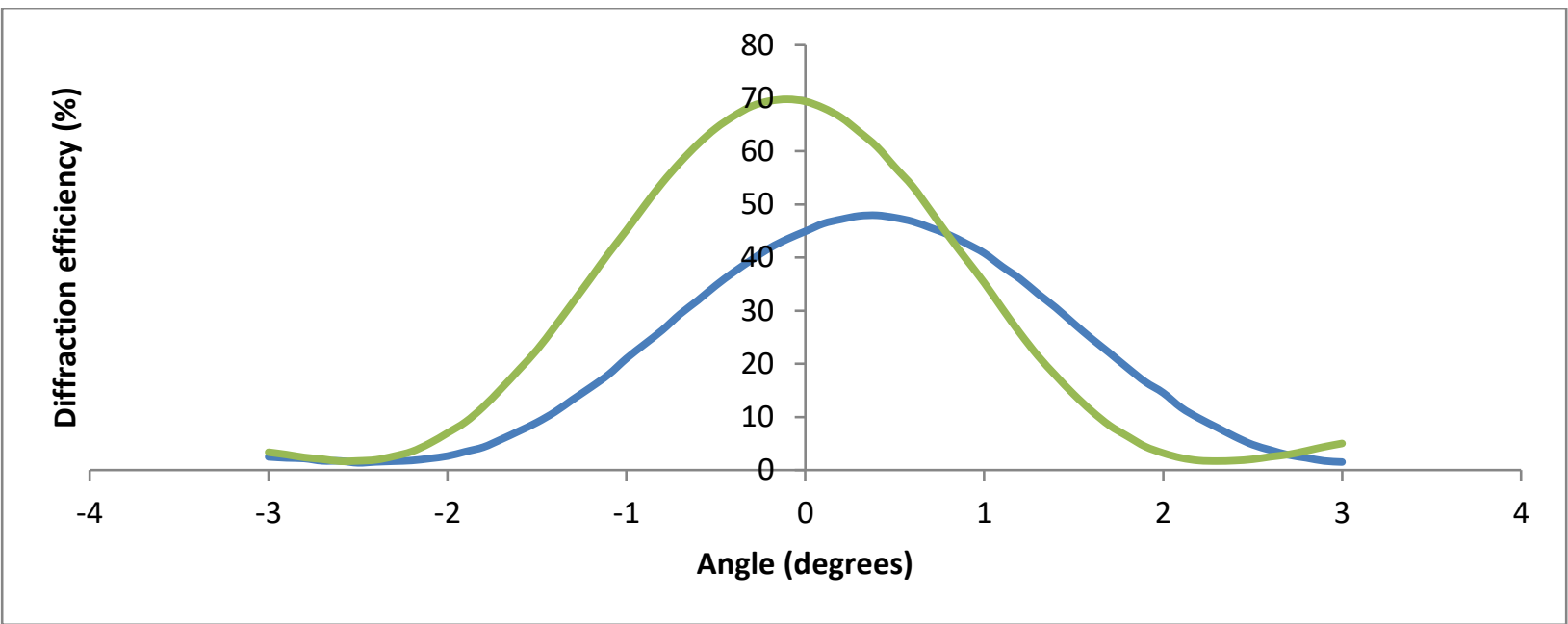

Figure 9: Bragg curves of MDEA sample coated in UV protective plastic before (green) and after (blue) 40 hours of UVA exposure.

\section{Conclusions:}

The optimisation of the acrylamide concentration led to an increase of refractive index modulation of $45 \%$. The rate of formation of the holographic gratings was also increased by a factor of 4.3 when compared to the standard formulation. When MDEA and TEA are compared at different thicknesses MDEA was shown to out-perform TEA as an initiator at higher thicknesses. Preliminary UV radiation tests show a decrease in diffraction efficiency when exposed at high intensities for long periods, thus further measures to stabilise the composition under UV exposure will need to be taken.

\section{Acknowledgements:}

Funding provided by Technological University Dublin’s Fiosraigh scholarship programme.

\section{References:}

[1] Vather, Dinesh; Naydenova, Izabela; Cody, Dervil; et al. "Serialized holography for brand protection and authentication” APPLIED OPTICS Volume: 57 Issue: 22 Pages: E131-E137 (2018)

[2] M. V. Collados, D. Chemisana, and J. Atencia, "Holographic solar energy systems: The role of optical elements,” Renew. Sustain. Energy Rev. 59, 130-140 (2016).

[3] G. Zhao, W. Li, J. Feng, G. Jiang, X. Wang, H. Liu "The effect of three electron donors on the initiator system efficiency of photopolymer film photosensitized by methylene blue". Materials and Design, 120 (2017), pp. 186-192

[4] Martin, S. (1995). "A new photopolymer recording material for holographic applications: photochemical and holographic studies towards an optimized system”. Pg 123 Doctoral thesis. Dublin Institute of Technology.

doi:10.21427/D7DP4B

[5] Moharam, M.G.; Young, L. “Criteria for Bragg and Raman-Nath regimes”. Appl. Opt. 1978, 17, 1757-1759.

[6] H. Kogelnik, "Coupled wave theory for thick hologram gratings" Bell Syst. Tech. J., 48 (9) (1969),

pp. 2909-2947 